\title{
A fuzzy goal programming approach to solve multi-objective supply chain network design problems
}

\author{
Amir Abbas Kanani Nezhad*, Emad Roghanian and Zahra Azadi
}

Department of Industrial Engineering, Khaje Nasir University of Technology, Tehran, Iran

\begin{tabular}{l}
\hline C H R O N I C L E \\
\hline Article history: \\
Received January 152012 \\
Received in revised format \\
April 162013 \\
Accepted April 162013 \\
Available online \\
April 172013 \\
\hline Keywords: \\
Logistics network \\
Fuzzy linear programming \\
Supply chain management
\end{tabular}
A B S T R A C T

The design of supply chain (SC) networks has attracted more attention in recent years according to business and environmental factors.in this paper a multi objective supply chain network design model aims to minimize network costs while satisfying the desired service level, is presented. A fuzzy goal programming (FGP) solution approach based on fuzzy membership function concept is developed to minimize costs and amount of investment while obtain maximum service level. Numerical experiments are conducted to test the efficiency of proposed solution method.

\section{Introduction}

The logistics network design has attracted much attention as companies have become more global, and more responsive to redesign distribution networks more frequently to operate at the lowest costs while providing the best customer service. A logistics network consists of suppliers, manufacturing centers, warehouses, distribution centers, and retail outlets as well as channels for the flow of raw materials, work-in-process inventory, and finished products between the facilities (Simchi-Levi et al., 1999). Logistics network design is associated with the determination of the number and location of warehouses and production plants, allocation of customer demand points to warehouses, and allocation of warehouses to production plants. The primary objective is to balance service level subject to various constraints such as production or purchasing costs, inventory holding and facility expenditures such as storage, handling and fixed costs and transportation costs. Therefore, it is essential to find a minimalannual-cost configuration of the distribution network, which satisfies product demands at specified customer service levels. Location problems are, in general, very difficult problems and the complexity increases as the number of customers, the number of products, the number of warehouses located and the number of potential locations for warehouses increase, which makes network design problems more

\footnotetext{
* Corresponding author. Tel: +989360005493

E-mail: amkaenani@gmail.com (A. A. Kanani Nezhad)

(C) 2013 Growing Science Ltd. All rights reserved. doi: $10.5267 /$ j.ijiec.2013.04.004
} 
complicated. To create an optimal network design/redesign, a 10-step logistics network design process is recommended:

1. Assess/evaluate current network,

2. Design and populate network optimization database,

3. Create network design alternatives, such as more or fewer hierarchies, multi-commodity flows, pooling opportunities, merge-in-transit, direct shipping, cross docking, and supply-flow optimization concepts,

4. Develop network optimization model,

5. Choose network optimization tool,

6. Implement network model in chosen tool,

7. Evaluate alternative network designs,

8. "Practicalize" recommended network structure,

9. Compute reconfiguration cost,

10. Make go/no-go decision.

\subsection{Literature review}

There have been many studies associated with deterministic supply chain network design problem where customer demand and other input parameters are given. Geoffrion and Graves (1974) studied a mixed integer linear programming for a multi-commodity capacitated single-period version of intermediate distribution facilities location and presented a new solution technique based on Benders Decomposition and examined their solution strategy for a major food firm with 17 commodity classes. Cohen and Lee (1998) presented a comprehensive model framework for linking decisions and performance throughout the material-production-distribution supply chain and introduced a heuristic optimization procedure. They also considered four sub modules to analyze interactions between functions in a SC network: (1) material control, (2) production control, (3) finished goods stockpile, and (4) distribution network control. Tragantalerngsak et al. (2000) studied two-echelon facility location problem where the number and the location of facilities in both echelons together with the allocation of customers to the second-echelon facilities were determined, simultaneously. They proposed a Lagrangian relaxation-based branch and bound algorithm for solving this problem. Jayaraman and Pirkul (2001) presented an integrated logistics model for locating production and distribution facilities in a multi-echelon environment. They provided a mixed integer programming formulation for the integrated model and provided an efficient heuristic solution procedure, which utilizes the solution generated from a Lagrangian relaxation of the problem.

Most articles consider forward and reverse flows separately, while reverse logistics activities influence on forward logistics such as warehouse capacity or vehicles. Beamon and Fernandes (2004) considered a closed-loop SC where used products are sent to manufacturers for remanufacturing and presented a multi-period integer programming model. Strategic decisions, which include logistic design, have longterm effects on company. In particular, they affect decisions around depot's number, location and size in long horizon. It causes a dynamic nature in logistic network, which result in large amount of uncertainties, which occur in customer's demand or in producing, distributing and dispensing and also collecting products. Stochastic programming is a way, which could help to model these uncertainties. Inderfuth et al. (2001) presented a periodic model for recovering products in a stochastic open shop. Listes and Dekker (2005) used a stochastic approach in a deterministic logistic network for recovering products. Santoso et al. (2005) presented a stochastic programming for logistic designing. They combined sample average approximation (SAA) approach with benders composition to solve the problem. Salema et al. (2007) proposed a reverse logistic model in which capacities constraint, multi product and stochastic demand were considered and developed a mixed linear programming to minimize total costs. However, the need of sufficient historical data that is rarely available in real-life cases and the high computational complexity are major drawbacks that make the use of stochastic 
programming models somehow impossible in real cases. Thus, a few number of works in recent years used more flexible approaches such as fuzzy and robust programming approaches.

Giannoccaro et al. (2003) proposed fuzzy echelon approach for inventory management where fuzzy set theory was used to properly model the uncertainty associated with both market demand and inventory costs. Guiffrida and Nagi (1998) gave a literature survey about fuzzy set theory applications in production management. In addition, in the framework of credibility theory, Yang et al. (2007) considered the logistics distribution centers location problem under fuzzy environment. They discussed distribution centers location problem under fuzzy environment and studied how to select distribution centers from the potential set so that the total relevant cost was minimized. In this paper, chance constrained programming model for the problem was designed and some properties of the model were investigated. Tabu search algorithm, genetic algorithm and fuzzy simulation algorithm were integrated to seek the approximate best solution of the model. Peidro et al. (2009) proposed a fuzzy mathematical programming model for supply chain planning which considered supply, demand and process uncertainties. The model has been formulated as a fuzzy mixed-integer linear programming model where data were ill-known and modeled by triangular fuzzy numbers. Qin and Ji (2010) employed a fuzzy programming tool to design the product recovery network. To solve the proposed models, they designed a hybrid intelligent algorithm, which integrated fuzzy simulation and genetic algorithm. Recently, Pishvaee and Razmi (2012) proposed a multi-objective fuzzy mathematical programming model for designing an environmental supply chain under inherent uncertainty of input data in such problem. The proposed model was capable to consider the minimization of multiple environmental impacts beside the traditional cost minimization objective to make a fair balance between them.

This paper is organized as follows. A multi objective logistic network design model is introduced in section 2. In section 3, fuzzy goal programming based solution approach is explained. The membership function construction method is illustrated in this section. Computational experiments are reported in section 4. Finally the conclusions of this paper and some directions for future researches are given in section 5 .

\section{Problem formulation}

In this section, the crisp formulation of the supply chain distribution network design model, originally introduced by Selim and Ozkarahan (2008), is presented. The goal of the model is to select the optimum numbers, locations and capacity levels of plants and warehouses to deliver the products to the retailers at the least cost while satisfying the desired service level to the retailers. Maximal covering approach is used in statement of the service level, and a coverage function, which may differ among the retailers according to their service standard request is defined for each retailer. The mathematical model is developed on the basis of the following assumptions:

-The network considered encompasses a set of retailers with known locations, and possible discrete set of location zones/sites where warehouses and plants are located.

-Different capacity levels are available to both the potential plants and warehouses.

- The retailers have demand for multitude of products, and the warehouses are responsible for righttime delivery of a right amount of products.

- Decision makers of the plants, warehouses and retailers share information and collaborate with each other to design an effective distribution network.

- Decisions are made within a single period.

\subsection{Notations and definitions}

Sets:

I: set of zones where retailers are located, 
$J$ : potential warehouse locations,

$K$ : potential plant locations,

$L$ : set of products,

$R$ : set of capacity levels available for warehouses,

$H$ : set of capacity levels available for plants.

Parameters:

$T_{j k l}$ : variable cost to transport one unit of product $l$ from the plant in zone $k$ to the warehouse in zone $j$,

$C_{i j l}$ : variable cost to transport one unit of product $l$ from the warehouse in zone $j$ to the retailer in zone $i$, $f_{k h}$ :fixed portion of the operating cost of the plant in zone $k$ with capacity level $h$

$g_{j r}$ : fixed portion of the annual possession and operating costs of the warehouse in zone $j$ with capacity level $r$, $O P_{k h}$ : opening cost of the plant in zone $k$ with capacity level $h$,

$O W_{j r}$ : opening cost of the warehouse in zone $j$ with capacity level $r$,

$a_{i l}$ : demand for product $l$ by the retailer in zone $i$,

$s_{l}$ : required throughput capacity of a warehouses for product $l$,

$W_{j r}$ : throughput capacity of the warehouse in zone $j$ with capacity level $r$,

$q_{l}$ : required production capacity of a plant for product $l$,

$D_{k h}$ : capacity of the plant in zone k with capacity level $h$,

$d_{t i j}$ :distance between zone $i$ and zone $j$,

Decision variables:

$Y_{j k l}$ : amount of product $l$ transported to the warehouse in zone $j$ from the plant in zone $k$,

$X_{i j l}$ : amount of product $l$ transported to the retailer in zone $i$ from the warehouse in zone $j$,

$Z_{j r}$; binary variable that indicates whether a warehouse with capacity level $r$ is constructed in zone $j$,

$P_{k h}$ : binary variable that indicates whether a plant with capacity level his constructed in zone $k$.

\subsection{Mathematical model}

$\min \sum_{i} \sum_{j} \sum_{l} C_{i j l} X_{i j l}+\sum_{j} \sum_{k} \sum_{l} T_{j k l} Y_{j k l}+\sum_{k} \sum_{h} f_{k h} P_{k h}+\sum_{j} \sum_{r} g_{j r} Z_{j r}$

$\min \sum_{k} \sum_{h} O P_{k h} P_{k h}+\sum_{j} \sum_{r} O W_{j r} Z_{j r}$

$\max \sum_{i} \sum_{j} \sum_{l} m_{i j} X_{i j l}$

subject to

$\sum_{i} X_{i j l}=a_{i l}$ for all $\mathrm{i} \in \mathrm{I}$ and $\mathrm{l} \in \mathrm{L}$,

$\sum_{i} \sum_{l} s_{l} X_{i j l} \leq \sum_{r} W_{j r} Z_{j r}$ for all $\mathrm{j} \in \mathrm{J}$,

$\sum_{r} Z_{j r} \leq 1$ for all $\mathrm{j} \in \mathrm{J}$,

$\sum_{i} X_{i j l} \leq \sum_{k} Y_{j k l}$ for all $\mathrm{j} \in \mathrm{J}$ and $\mathrm{l} \in L$,

$\sum_{j} \sum_{l} q_{k l} Y_{j k l} \leq \sum_{h} D_{k h} P_{k h}$ for all $\mathrm{k} \in \mathrm{K}$,

$\sum_{i} P_{k h} \leq 1$ for all $\mathrm{k} \in \mathrm{K}$,

$Z_{j r} \in\{0,1\}$ for all $\mathrm{j} \in \mathrm{J}, \mathrm{r} \in R, \mathrm{P}_{k h} \in\{0,1\}$ for all $\mathrm{k} \in K, \mathrm{~h} \in H$. 
The first objective is to minimize total costs (TCOST) of products from plants to warehouses and from warehouses to retailers + fixed costs associated with the plants and the warehouses. The second one minimizes investment (INV) in opening plants and warehouses and finally the last objective maximizes total service level (TSERVL) provided to the retailers. Constraint set (4) ensures that all demand from retailers is satisfied by warehouses. Constraint set (5) limits the distribution quantities that are shipped from warehouses to retailers to the throughput limits of warehouses. Constraint set (6) ensures that a warehouse can be assigned at most one capacity level. Constraint set (7) guarantees that all demand from retailer in zone $i$ for product $l$ is balanced by the total units of product 1 available at warehouse in zone $j$ that has been supplied from open plants. Constraints in set (8) represent the capacity restrictions of the plants in terms of their total shipments to the warehouses. Constraint set (9) ensures that a plant can be assigned at most one capacity level. Finally, constraint set (10) enforces the binary and nonnegative restrictions on the decision variables.

Selim and Ozkarahan (2008) used the "fuzzy and" operator to deal with multi-objective issue of problem. By this way, they obtain a category of compromise solutions that Decision Maker (DM) can choose the best one between them according to supposable aspiration level of each goal in problem. However, we use fuzzy goal programming approach to solve the problem stated in this paper In order to obtain a unique optimal solution and make a framework to build a lower and upper bound for the problem solution. It helps DM to acquire a unique optimal solution that would be the best one due to contribution of fuzzy goal programming method for solving multi-objective problems. Also, DM can build other solutions of problem by changing the upper and lower limit of objective functions in construction of fuzzy membership functions. The advantage of our proposed method is simplicity and its strength to obtain a unique optimal solution according to DM's preference.

\section{The proposed fuzzy goal programming solution approach}

An approach to solve the multi objective problems is fuzzy goal programming. In this manner, for each objective, a membership function is constructed. Then these fuzzy objectives are termed as fuzzy goals which mean the aspiration level of each objective. Finally, in order to minimize the deviations of maximum aspiration level, that is one for fuzzy membership functions, goal programming is used.

\subsection{Construction of fuzzy membership functions}

In order to change the objective functions to fuzzy membership functions, fuzzy LR functions are utilized.in this way, an upper and lower bound should be assigned for each objective function. A solution set $\mathrm{X}$ is obtained for FGP that let $\mathrm{g}_{\mathrm{k}}$ be the aspiration level of kth objective, $\mathrm{f}_{\mathrm{k}}(\mathrm{X})$ $(\mathrm{k}=1,2, \ldots, \mathrm{n})$. Then, the FGP can be expressed as the following form:

$f_{k}(X) \succsim g_{k}\left(\right.$ or $\left.f_{k}(X) \precsim g_{k}\right), \quad k=1,2, \ldots, M$

subject to $X \in F, \quad$ (F is a feasible set),

where $f_{k}(X) \gtrsim(\precsim) g_{k}$ indicates the $\mathrm{k}^{\text {th }}$ fuzzy goal approximately greater than or equal to (approximately less than or equal to) the aspiration level $g_{k}$; other variables are defined as in GP. $\mu_{k}\left(f_{k}(X)\right)$ is a membership function of the $k^{\text {th }}$ objective. The FGP has the advantage of allowing for the vague aspirations of a DM, which can be qualified using some natural language or vague phenomena. For representing the preference concept from DMs, $\mu_{k}\left(f_{k}(X)\right)$ is then characterized as follows:

For maximization-type objectives:

$$
\mu_{k}\left(f_{k}(X)\right)= \begin{cases}1 & \text { if } \mathrm{f}_{k}(X) \geq g_{k}, \\ \frac{\left(f_{k}(X)-l_{k}\right)}{g_{k}-l_{k}}, & \text { if } \mathrm{l}_{k} \leq f_{k}(X) \leq g_{k} \text { for } \mathrm{f}_{k}(X) \geq g_{k}, \\ 0 & \text { if } \mathrm{f}_{k}(X) \geq l_{k} .\end{cases}
$$


For minimization-type objectives:

$$
\mu_{k}\left(f_{k}(X)\right)= \begin{cases}1 & \text { if } \mathrm{f}_{k}(X) \geq g_{k}, \\ \frac{\left(u_{k}-f_{k}(X)\right)}{u_{k}-g_{k}}, & \text { if } \mathrm{g}_{k} \leq f_{k}(X) \leq u_{k} \text { for } \mathrm{f}_{k}(X) \leq g_{k}, \\ 0 & \text { if } \mathrm{f}_{k}(X) \geq u_{k}\end{cases}
$$

where $l_{k}$ and $u_{k}$ are, respectively, lower and upper limits for the $k^{\text {th }}$ goal; $f_{k}(X)$ and $g_{k}$ are defined as in GP. The membership function for minimization-type objectives is shown in Fig.1. The aspiration levels are determined by DMs. In addition to the aspiration levels of the goals, FGP needs max-min limits $\left(u_{k}\right.$, $l_{k}$ ) for each goal. While the DMs decide the maxmin limits, the linear programming results are starting points and the intervals are covered by these results. Generally, the DMs find estimates of the upper $(u)$ and lower $(l)$ values for each goal using payoff table (see Table 1). Therefore, the feasibility of each fuzzy goal is guaranteed. Here, $Z_{m}(X)$ denotes the $m^{\text {th }}$ objective function, and $X(m)$ is the optimal solution of the $m^{\text {th }}$ single objective problem. Solving the problem with $X(m)(m=1, \ldots, M)$ for each objective, a payoff matrix with entries $Z_{p m}=Z_{m}(X(P)), m, p=1, \ldots, M$ can be formulated as presented in Table 1. Here, for minimization-type objectives, $\mathrm{u}_{\mathrm{m}}=\max \left(Z_{1 m}, Z_{2 m}, \ldots, Z_{M m}\right)$ and $g_{m}=Z_{m m}$, $m=1, \ldots, M$.

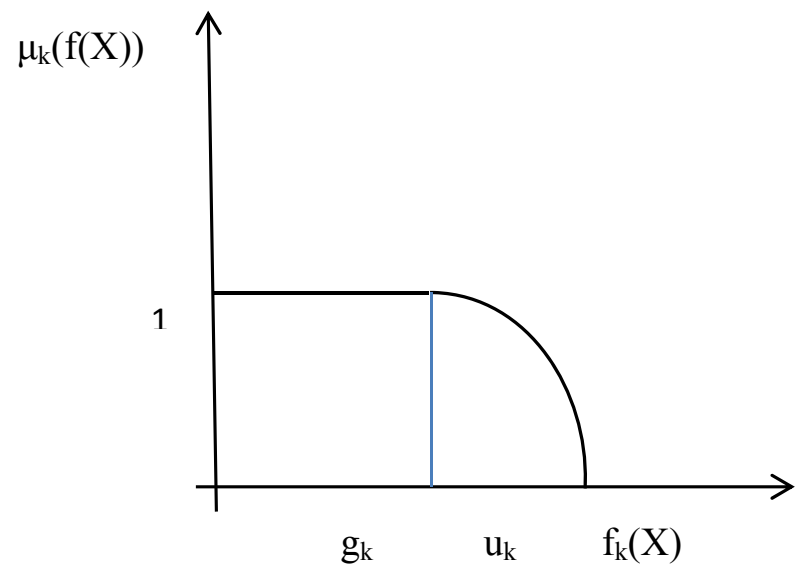

Fig. 1. Membership function for minimization-type objectives

Table 1

Payoff table

\begin{tabular}{ccccc}
\hline & $\mathrm{Z}_{1}(\mathrm{X})$ & $\mathrm{Z}_{2}(\mathrm{X})$ & $\ldots$ & $\mathrm{Z}_{\mathrm{M}}(\mathrm{X})$ \\
\hline $\mathrm{X}^{(1)}$ & $\mathrm{Z}_{11}$ & $\mathrm{Z}_{12}$ & $\ldots$ & $\mathrm{Z}_{1 \mathrm{M}}$ \\
$\mathrm{X}^{(2)}$ & $\mathrm{Z}_{21}$ & $\mathrm{Z}_{22}$ & $\ldots$ & $\mathrm{Z}_{2 \mathrm{M}}$ \\
$\vdots$ & $\ldots$ & $\ldots$ & $\ldots$ & $\ldots$ \\
$\mathrm{X}^{(\mathrm{M})}$ & $\mathrm{Z}_{\mathrm{M} 1}$ & $\mathrm{Z}_{\mathrm{M} 2}$ & $\ldots$ & $\mathrm{Z}_{\mathrm{MM}}$ \\
\hline
\end{tabular}

\subsection{Fuzzy goal programming solution approach}

Goal programming (GP) is an important technique for decision-makers (DM) to consider simultaneously the conflicting objectives in finding a set of acceptable solutions. It can be said that GP has been still the most widely used technique for solving multi-criteria and multi-objective decision making problems. In a conventional GP formulation, goals are precisely defined. That is, the formulation assumes that the DM is able to determine accurately goal values for their decision-making problems. In fact, many imprecise aspiration levels may exist in decision-making problems such as "somewhat larger than", "substantially lesser than", or "around" the vague goal $g_{k}$ due to DM's ambiguous understanding of their nature. Thus, the DM may find it is impossible to state precisely exact aspiration levels to the goals for their problems. In doing so, if the imprecise aspiration level is 
introduced to each objective, then the problem is turned into fuzzy GP (FGP) (Chen \& Tsai, 2001; Giannoccaro et al., 2003; Listes, 2005; Mohamed, 1997; Narasimhan, 1980; Tjendera Santoso et al., 2005; Lai \& Hwang, 1994). Since Narasinhan (1980) applied the fuzzy set theory with preference based membership function to GP, many achievements have been reported in the literature such as preemptive FGP, weight additive model, stochastic model, and real-life case studies (Chen \& Tsai, 2001). Zimmermann (1978) first proposed fuzzy programming for solving the multi objective linear programming problems. Narasimhan (1980) presented the initial FGP model and solution procedure. Hannan (1981) introduced interpolated membership functions (i.e., piecewise linear membership functions) into the FGP model, then the FGP model could be solved using the linear programming method. However, Hannan's model is applicable only to FGP problems with concave membership functions. To further improve the model of Hannan, Yang et al. (2007) formulated the FGP problem using fewer additional variables. The concept of different importance and priority use in FGP was considered by Chen and Tsai (2001). A typical FGP can be expressed as follows:

In fuzzy programming approaches, the highest degree of membership function is 1 . So, for the defined membership functions in (11) and (12), the flexible membership goals with the aspired level 1 can be presented as

$\mu_{k}\left(f_{k}(X)\right)+d_{k^{-}}-d_{k^{+}}=1 ; \mathrm{k}=1,2, \ldots, \mathrm{M}$

or equivalently as:

$\frac{\left(u_{k}-f_{k}(X)\right)}{u_{k}-g_{k}}+\mathrm{d}_{\mathrm{k}}{ }^{-}-\mathrm{d}_{\mathrm{k}}{ }^{+}=1$ (for minimization-type objectives)

where $d_{k}^{-}, d_{k}^{+} \geq 0$ and $d_{k}^{-} \times d_{k}^{+}=0$ represent the under deviation and over deviation, respectively, from the aspired levels. In conventional GP, the under- and/or over deviational variables are included in the achievement function for minimizing them and that depend upon the type of the objective functions to be optimized. In this approach, the over-deviational variables for the fuzzy goals of objective functions, $d_{k}$, required to be minimized to achieve the aspired levels of the fuzzy goals. Note that any under-deviation from a fuzzy goal indicates the full achievement of the membership value. Consequently, with use of fuzzy goal programming approach, the multi objective linear programming can be presented as below:

$\min \mathrm{Z}=\sum_{k=1}^{M} W_{k} \times d_{k}^{+}$

subject to

$\frac{\left(u_{k}-f_{k}(X)\right)}{u_{k}-g_{k}}+d_{k^{-}}-d_{k^{+}}=1 ; \mathrm{k}=1, \ldots, \mathrm{M}$ (for minimization-type objectives)

$\frac{\left(f_{k}(X)-l_{k}\right)}{g_{k}-l_{k}}+d_{k^{-}}-d_{k^{+}}=1 ; \mathrm{k}=1, \ldots, \mathrm{M}$ (for maximization-type objectives)

$\mathrm{A} \times \mathrm{X}<$ or $=$ or $>\mathrm{b} \quad$ (technical constraints of problem)

$\mathrm{d}_{k^{-}}, \mathrm{d}_{k^{+}} \geq 0$ with $\mathrm{d}_{k^{-}} \times \mathrm{d}_{k^{+}}=0$

It should be noted that, $\mathrm{W}_{\mathrm{k}}$ is the importance weight for $\mathrm{k}_{\text {th }}$ objective function and it is obtained by DM attitude.

\section{Numerical example}

To illustrate the fuzzy goal programming solution approach, computational experiments are presented in this section. The model is solved using Lingo 11 optimization software. A hypothetically constructed SC distribution network design problem with 2 retailer zones, 2 potential warehouse sites and 2 
potential plant sites is considered in this example. It is assumed that two different types of product are demanded by retailers. The parameters of the problem are supposed to be as below:

Expected demands of the retailers for two different products are drawn from a uniform distribution between 100 and 1000 as given in Table 2. Five capacity levels are used for the capacities available to both the potential plants and warehouses. The opening cost of the warehouse in zone $j$ with capacity level $3\left(\mathrm{OW}_{\mathrm{j} 3}\right)$ are drawn from a uniform distribution between 90000 and 120000 . The opening costs of the warehouses for the other capacity levels are assumed to be: $\mathrm{OW}_{\mathrm{j} 1}=.75 \times \mathrm{OW}_{\mathrm{j} 3}, \mathrm{OW}_{\mathrm{j} 2}=.85 \times \mathrm{OW}_{\mathrm{j} 2}$, $\mathrm{OW}_{\mathrm{j} 4}=1.15 \times \mathrm{OW}_{\mathrm{j} 3}, \mathrm{OW}_{\mathrm{j} 5}=1.25 \times \mathrm{OW}_{\mathrm{j} 3}$. Cost coefficients of $\mathrm{OP}_{\mathrm{kh}}$ are computed in terms of the warehouses costs as $\mathrm{OP}_{\mathrm{kh}}=4 \times \mathrm{OW}_{\mathrm{kh}}$. Fixed portion of the annual possession and operating costs of the warehouse in zone $j$ with capacity level $3\left(\mathrm{~g}_{\mathrm{j} 3}\right)$ and the plant in zone k with capacity level $3\left(\mathrm{f}_{\mathrm{k} 3}\right)$ are drawn from a uniform distribution between 18,000 and 25,000 and 75,000 and 100,000, respectively. Fixed portion of the annual possession and operating costs of warehouses and plants for the other capacity levels are computed as follows: $\mathrm{g}_{\mathrm{j} 1}=0.75 \times \mathrm{g}_{\mathrm{j} 3}, \mathrm{~g}_{\mathrm{j} 2}=0.85 \times \mathrm{g}_{\mathrm{j} 3}, \mathrm{~g}_{\mathrm{j} 4}=1.15 \times \mathrm{g}_{\mathrm{j} 3}, \mathrm{~g}_{\mathrm{j} 5}=1.25 \times \mathrm{g}_{\mathrm{j} 3}$ and $\mathrm{f}_{\mathrm{k} 1}=0.75 \times \mathrm{f}_{\mathrm{k} 3}, \mathrm{f}_{\mathrm{k} 2}=0.85 \times \mathrm{f}_{\mathrm{k} 3}, \mathrm{f}_{\mathrm{k} 4}=1.15 \times \mathrm{f}_{\mathrm{k} 3}, \mathrm{f}_{\mathrm{k} 5}=1.25 \times \mathrm{f}_{\mathrm{k} 3}$.

Required throughput capacity of a warehouse for product 1 and required production capacity of a plant for product 1 are given as follows: $\mathrm{s}_{1}=1, \mathrm{~s}_{2}=1$ and $\mathrm{q}_{1}=1, \mathrm{q}_{2}=2$. The cost coefficients $\mathrm{C}_{\mathrm{ij} 1}$ and $\mathrm{T}_{\mathrm{jk} 1}$ are given as below:

$\mathrm{C}_{\mathrm{ij} \mathrm{l}}=12,11,14,15,24,10,10.5,36$ and $\mathrm{T}_{\mathrm{jk} \mathrm{l}}=12,15,16,20,21,14,15,17$.

Throughput limit of warehouse in zone $\mathrm{j}$ with capacity level $\mathrm{r}\left(\mathrm{W}_{\mathrm{jr}}\right)$ and capacity of the plant in zone $\mathrm{k}$ with capacity level $h\left(D_{\text {kh }}\right)$ are taken as follows.

$\mathrm{W}_{\mathrm{jr}}=4000,6000,8000,10000,12000, \mathrm{D}_{\mathrm{kh}}=15000,20000,30000,35000,40000$.

\subsection{Solution by proposed approach}

Step1.The efficient extreme solution of the problem is obtained by solving each objective of model in section III under restrictions of problem constraints. The payoff table (Table 3) is constructed by putting each objective optimal solution that calculated in isolation in other objective and calculating its amount.

Table 2

Expected demand of the retailers

\begin{tabular}{rrrrr}
\hline \multicolumn{2}{c}{ Demand for product 1 } & \multicolumn{2}{c}{ Demand for product 2 } \\
\hline 992 & 408 & 229 & 282 \\
\hline
\end{tabular}

Table 3

Payoff table

\begin{tabular}{cccc}
\hline & TCOST & INV & TSERVL \\
\hline TCOST & 137681.8 & 222219.8 & 553.6500 \\
INV & 170208.6 & 115708.4 & 553.6500 \\
TSERVL & 358851.5 & 590178.8 & 675.75 \\
\hline
\end{tabular}

Step 2.According to payoff table in previous step and by means of the membership function construction method that explained in section IV, membership function for each objective of problem is constructed. 


$$
\begin{aligned}
& \mu_{T \text { cost }}= \begin{cases}1 & \text { if } \mathrm{f}_{T \cos t} \leq 137681.8, \\
\frac{(358851.5-T \cos t)}{358851.5-137681.8}, & \text { if } 137681.8 \leq T \cos t \leq 358851.5 \text { for } \mathrm{f}_{k}(X) \prec g_{k}, \\
0, & \text { if } T \cos t \geq 358851.5\end{cases} \\
& \mu_{I N V}= \begin{cases}1 & \text { if } \mathrm{f}_{I N V} \leq 115708.4, \\
\frac{(590178.8-I N V)}{590178.8-115708.4}, & \text { if } 115708.4 \leq I N V \leq 590178.8 \text { for } \mathrm{f}_{k}(X) \prec g_{k}, \\
0, & \text { if } I N V \geq 590178.8\end{cases}
\end{aligned}
$$$$
\mu_{\text {TSERVL }}= \begin{cases}1 & \text { if } \mathrm{f}_{\text {ITSERVL }} \leq 675.75, \\ \frac{(T S E R V L-553.65)}{675.75-553.65}, & \text { if } 553.65 \leq \text { TSERVL } \leq 675.75 \text { for } \mathrm{f}_{k}(X) \succ g_{k}, \\ 0, & \text { if } T S E R V L \geq 553.65\end{cases}
$$

Step 3.According to approach explained in section IV and the membership functions obtained from previous step, the fuzzy goal programming model is developed and is optimized by lingo software. The results are summarized in Table 4.

\section{Table 4}

Solution results of the model by the proposed solution approach

\begin{tabular}{ccccccccc}
\hline TCOST & INV & TSERVL & \multicolumn{2}{c}{$\boldsymbol{\mu}_{\text {Tcost }}$} & \multicolumn{2}{c}{$\boldsymbol{\mu}_{\text {INV }}$} & \multicolumn{2}{c}{$\boldsymbol{\mu}_{\text {TSERVL }}$} \\
\hline 83239.9052 & 32987.19 & 675.75 & O.D $^{\mathrm{a}}$ & U.D $^{\mathrm{b}}$ & O.D & U.D & O.D & U.D \\
\hline & deviations & & 0.2461544 & 0.0000000 & 0.1743443 & 0.0000000 & 0.0000000 & 0.0000000 \\
\hline
\end{tabular}

${ }^{\mathrm{a}}$ Over Deviation ${ }^{\mathrm{b}}$ Under Deviation

\section{Conclusion}

Designing supply chain network has attracted many attentions because of its importance nowadays. In this paper, a supply chain network design model was introduced and a fuzzy goal programming solution approach was developed to minimize total costs. The proposed model contained transportation and fixed costs of opening facilities and amount of investment while satisfying desired level of service to dispel costumers demand. To cope crisp environment of model to fuzzy goal programming environment, fuzzy membership function concept has been utilized and to the best of our knowledge, the developed method is one of the simplest methods to solve multi objective problems especially for supply chain network design models.

Finally, there are some possible directions for future research. Since uncertainty is one of the impartible concepts of all network designing problems, importing it to these problems by using fuzzy theory and combining it by developed solution method in this paper can be considered. Some other solution methods based on fuzzy membership function to deal with multi objective problems can be developed. Moreover, the simple model of this paper can be developed by adding more objectives such as qualitative objectives and some other constraints such as reverse flow of material in network and designing closed-loop networks.

\section{References}

Beamon, B. M., \& Fernandes, C. (2004). Supply-chain network configuration for product recovery. Production Planning \& Control, 15(3), 270-281.

Chen, L. H., \& Tsai, F. C. (2001). Fuzzy goal programming with different importance and priorities. European Journal of Operational Research, 133(3), 548-556. 
Cohen, M. A., \& Lee, H. L. (1988). Strategic analysis of integrated production-distribution systems: models and methods. Operations Research, 36(2), 216-228.

Geoffrion, A. M., \& Graves, G. W. (1974). Multicommodity distribution system design by Benders decomposition. Management science, 20(5), 822-844.

Giannoccaro, I., Pontrandolfo, P., \& Scozzi, B. (2003). A fuzzy echelon approach for inventory management in supply chains. European Journal of Operational Research, 149(1), 185-196.

Guiffrida, A. L., \& Nagi, R. (1998). Fuzzy set theory applications in production management research: a literature survey. Journal of intelligent manufacturing,9(1), 39-56.

Selim, H., \& Ozkarahan, I. (2008). A supply chain distribution network design model: An interactive fuzzy goal programming-based solution approach, International Journal of Advanced Manufacturing Technology, 36,401-418.

Hannan, E. L. (1981). ON FUZZY GOAL PROGRAMMING*. Decision Sciences, 12(3), 522-531.

Inderfurth, K., de Kok, A. G., \& Flapper, S. D. P. (2001). Product recovery in stochastic remanufacturing systems with multiple reuse options. European Journal of Operational Research, 133(1), 130-152.

Jayaraman, V., \& Pirkul, H. (2001). Planning and coordination of production and distribution facilities for multiple commodities. European journal of operational research, 133(2), 394-408.

Lai, Y. J., \& Hwang, C. L. (1994). Fuzzy Multiple Objective Decision Making: Methods and Applications.

Listeş, O., \& Dekker, R. (2005). A stochastic approach to a case study for product recovery network design. European Journal of Operational Research, 160(1), 268-287.

Mohamed, R. H. (1997). The relationship between goal programming and fuzzy programming. Fuzzy Sets and Systems, 89(2), 215-222.

Narasimhan, R. (1980). Goal programming in a fuzzy environment. Decision sciences, 11(2), 325-336.

Peidro, D., Mula, J., Poler, R., \& Verdegay, J. L. (2009). Fuzzy optimization for supply chain planning under supply, demand and process uncertainties. Fuzzy Sets and Systems, 160(18), 2640-2657.

Pishvaee, M. S., \& Razmi, J. (2012). Environmental supply chain network design using multi-objective fuzzy mathematical programming. Applied Mathematical Modelling, 36(8), 3433-3446.

Qin, Z., \& Ji, X. (2010). Logistics network design for product recovery in fuzzy environment. European Journal of Operational Research, 202(2), 479-490.

Salema, M. I. G., Barbosa-Povoa, A. P., \& Novais, A. Q. (2007). An optimization model for the design of a capacitated multi-product reverse logistics network with uncertainty. European Journal of Operational Research,179(3), 1063-1077.

Santoso, T., Ahmed, S., Goetschalckx, M., \& Shapiro, A. (2005). A stochastic programming approach for supply chain network design under uncertainty. European Journal of Operational Research, 167(1), 96-115.

Simchi-Levi, D., Simchi-Levi, E., \& Kaminsky, P. (1999). Designing and managing the supply chain: Concepts, strategies, and cases. United-States: McGraw-Hill.

Tragantalerngsak, S., Holt, J., \& Rönnqvist, M. (2000). An exact method for the two-echelon, singlesource, capacitated facility location problem. European Journal of Operational Research, 123(3), 473-489.

Yang, L., Ji, X., Gao, Z., \& Li, K. (2007). Logistics distribution centers location problem and algorithm under fuzzy environment. Journal of Computational and Applied Mathematics, 208(2), 303-315.

Zimmermann, H. J. (1978). Fuzzy programming and linear programming with several objective functions. Fuzzy sets and systems, 1(1), 45-55. 